\title{
Methods and technologies of seismic strengthening of buildings with brick walls
}

\author{
Vladimir Kondratiev ${ }^{1 *}$, Hamza Yusupov $^{2}$, Vera Egorova $^{1}$, and Said Kurtametov ${ }^{1}$ \\ ${ }^{1}$ Samarkand State Architectural and Civil Engineering Institute, 140147, Samarkand, Uzbekistan \\ ${ }^{2}$ Tashkent Institute of Architecture and Civil Engineering, 100011, Tashkent, Uzbekistan
}

\begin{abstract}
Methods and instructions on the production technologies for the restoration and reinforcement of buildings with walls made of fired bricks, which have received various damages as a result of earthquakes, physical wear, and also have inconsistencies in space-planning and design solutions with the requirements of current regulatory documents, are given.

The most typical types of damage to elements and structures of buildings as a result of earthquakes were identified and classified according to the results of analyzes of their consequences, in which the authors were directly involved (by the nature of their scientific and practical activities), as well as according to the analytical review of the relevant sources.

The identification and compilation of classifications of the most common facts of inconsistency of space-planning and structural solutions with the requirements of the current design standards was carried out on the basis of the results of field inspections of the technical condition of numerous objects, the experience of which was accumulated by the authors over the past several years.

Based on the results obtained, methods and technologies for seismic amplification of elements and structures of buildings have been developed, reflecting their features, composition and sequence of operations for the production of work, also based on the authors' many years of experience in the implementation of similar developments, acquired during the implementation of a number of state scientific and technical programs, innovative projects and contractual work.

For each of the considered cases and reinforcement options, the corresponding standards have been developed in relation to the labor intensity and cost of performing work, the recommended composition of the links and the corresponding standard set.
\end{abstract}

\section{Introduction}

Due to the peculiarities of its geographical location, the main part of the territory of the Republic of Uzbekistan is subject to the danger of emergencies, both natural and manmadeones, which can be caused by earthquakes that are the most formidable natural phenomena and the most dangerous in terms of social and economic damage.

\footnotetext{
*Corresponding author: vkondratev1958@mail.ru
} 
Earthquakes have long been a serious threat to the population and national economy of Uzbekistan. About half of the republic's territory can be subject to earthquakes with an intensity of 7 points and higher. More than 20 million people live in this zone.There are more than 330 settlements, including 120 cities. According to the "Catalog of Earthquakes", earthquakes with a magnitude of up to 7.5 can occur on the territory of Uzbekistan. All this indicates that for Uzbekistan, the danger of earthquakes, as well as all other accompanying and provoked emergencies, is quite high.

The authors of the paper, by the nature of their scientific and practical activities, were directly involved in the analysis and study of the consequences of a number of earthquakes that occurred in the territory of the republic over the last more than half a century, including the facts of historical seismicity. A huge amount of factual material has been accumulated on the results of field surveys of the technical condition of buildings, ensuring their seismic safety, the development of measures and projects for their seismic enhancement, implemented within the framework of state scientific and technical programs, the implementation of innovative projects and developments, as well as contractual work.

In particular, according to the results of the inventory of the housing stock of Samarkand and the certification of buildings, it was found that buildings with walls made of fired bricks have a rather significant proportion (they contain about $35 \%$ of the city's living space).

Buildings of the noted design solution with the number of floors from 2 to 5 , erected in the 50-60s of the last century (and in a later period), do not meet the requirements of the current building code (KMK) [1] for a number of parameters (length, height, number of floors, parameters of the openings, step of the transverse walls, presence of wall reinforcement elements, etc.) and reached a rather significant level of physical wear. The total number of such buildings is about $55 \%$.

Along with the intensively ongoing reconstruction of cities in the republic, the construction of modern comfortable housing, including in rural areas, the buildings of the above-mentioned design solutions are currently in operation. Taking into account the existing realities and forecasts, it seems that this trend will continue for quite a long time. It should also be noted that a similar situation is typical for other cities of the republic.

In this regard, the problem of ensuring the seismic and operational safety of such buildings and the ensuing tasks of carrying out a set of preventive measures seem to be vital and extremely urgent.

Among the set of measures that make up a strategy for reducing seismic risk, a special place is taken by the tasks of developing methods and technologies for seismic strengthening of buildings, which is one of the subjects of the authors' research, the coverage of individual results of which is the purpose of this paper.

\section{Materials and methods}

The research consisted in identifying and classifying the most common facts of inconsistency of the space-planning and structural solutions of buildings with walls made of fired bricks with the requirements of the current design standards (KMK) [1], which were recorded as a result of field examinations of the technical state of numerous objects, the experience of which has been accumulated by the authors over the past several years. Similar classifications were drawn up in relation to the most typical types of damage to elements and structures of buildings as a result of earthquakes, revealed by the results of the analysis of their consequences directly with the participation of the authors, as well as according to the analytical review of the relevant sources.

On the basis of the results obtained, methods and technologies for seismic strengthening of elements and structures of buildings were developed, reflecting their features, 
composition and sequence of operations for the production of work, also based on the authors' many years of experience in the implementation of similar developments, acquired during the execution of a number of state scientific and technical programs, innovation projects and economic and commercial works.

For each of the considered cases and reinforcement options, the corresponding standards have been developed in relation to the labor intensity and cost of performing work, the recommended composition of the links and the corresponding standard set. Due to the fact that specialized regulatory documents (ShHK) regulating the issues of technologies for strengthening structures have not yet been developed in Uzbekistan, these standards have been drawn up on the basis of an appropriate selectionoftypes of processes and works, which aresuitable and similar in nature and meaning, regulated by the current domestic ShHK for repair and other types of work [2-4, etc.], as well as the corresponding selection from foreign regulatory documents.

\section{Strengthening of separation walls}

\section{Separation walls with diagonal and $x$-shapedcracks.}

Area of application. Narrow and high walls with dimensions that do not meet the requirements of the building code [1], with diagonal and x-shaped cracks (Fig. 1).

List of technological operations:

1. Plaster is removed from both sides of the wall;

2. A welded frame is made from corners $50 \times 50 \times 5$ and strip steel $50 \times 5$, with dimensions along the width and thickness of the wall;

3. Installed frames are pulled together.Steel plates $50 \times 5 \mathrm{~mm}$ are welded to themat $50 \mathrm{~cm}$ in height, passed between the wall and the frame. After welding, the screeds are removed;

4. The separation wall is wrapped with a mesh attached with annealed wire to the frame elements;

5. After moistening the masonry, the separation wall is plastered with M-50 mortar.

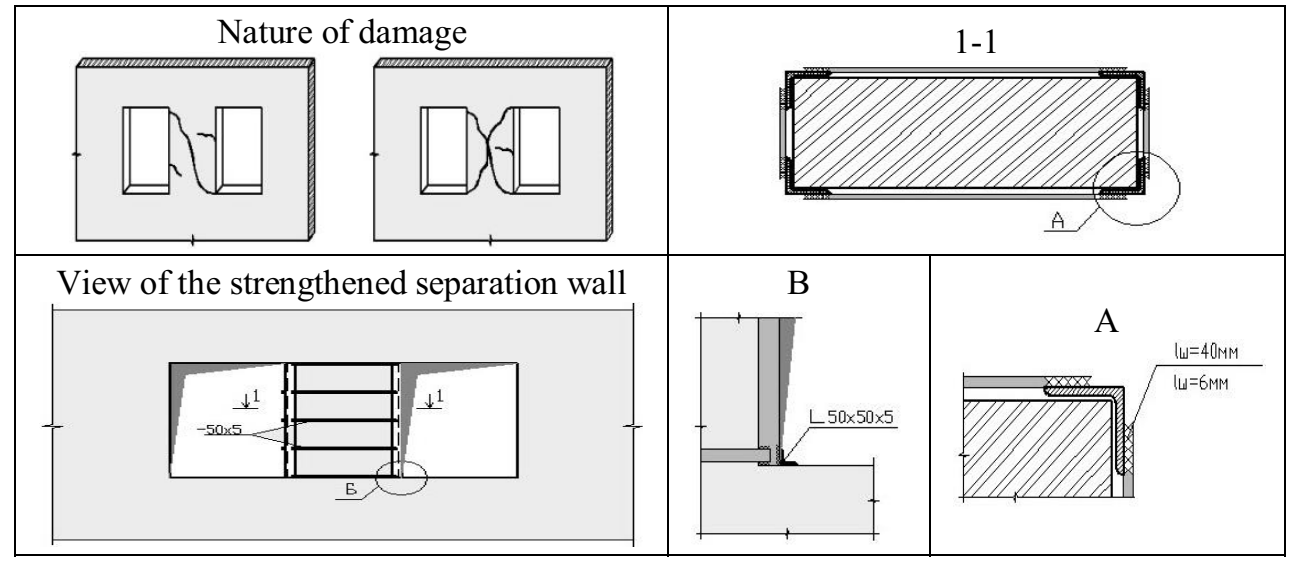

Fig. 1. Strengthening of narrow and high separation walls, with dimensions that do not meet the requirements of the building code [1], with diagonal and $\mathrm{x}$-shaped cracks.

An alternative is the option of strengthening such separation walls by installing a double-sided reinforced cement-sand cage with grids (Fig. 2) 
List of technological operations:

1. Plaster is removed from both sides of the wall;

2. The surface is cleaned of plaster residues with clearing of the joints to a depth of 1.0-1.5 $\mathrm{cm}$;

3. The cleaned surface is rinsed with water under pressure;

4. Holes are made between the frame and the wall through $50 \mathrm{~cm}$ along the height of the wall;

5. A grid with a mesh size of $150 \times 150 \mathrm{~mm}$ from a wire with a diameter of $5 \mathrm{~mm}$ is installed on both sides of the wall;

6. In the corners of the separation wall against the holes, reinforcement with a diameter of $6 \mathrm{~mm}$ of class A-I is installed and fastened to the grids. Along the height of the wall, every $50 \mathrm{~cm}$ in a staggered manner, holes are drilled for the passage of Z-shaped ties from reinforcement with a diameter of $6 \mathrm{~mm}$ of class A-I;

7. Connecting elements made of steel of class A-I with a diameter of $6 \mathrm{~mm}$ are passed through the holes and welded with gussets. Weld seam length is $50 \mathrm{~mm}$;

8. A layer of plaster with a thickness of $3-4 \mathrm{~cm}$ is applied on the grid by the shot creting method.

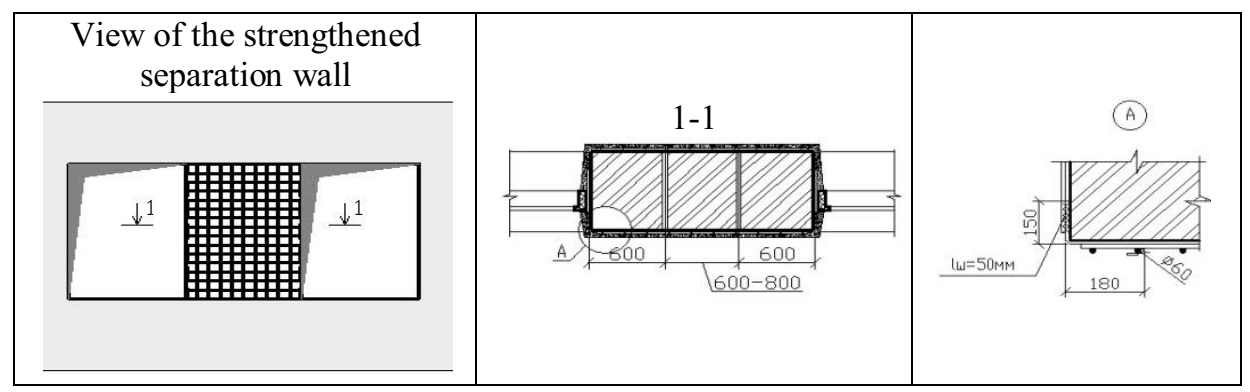

Fig. 2. Strengthening of separation walls with a width that does not meet the requirements of the KMK [1], with diagonal and X-shaped cracks.

\section{Separation walls with horizontal cracks}

Area of application. Narrow and high separation walls with dimensions that do not meet the requirements of the building code [1], with horizontal, diagonal and X-shapedcracks (Fig. $3)$.

List of technological operations:

1. Plaster is removed from both sides of the wall;

2. A welded frame is made from corners $50 \times 50 \times 5$ and strip steel $50 \times 5$, with dimensions along the width and thickness of the wall;

3. Installed frames are pulled together.Steel plates $50 \times 5 \mathrm{~mm}$ are welded to them at $50 \mathrm{~cm}$ in height, passed between the wall and the frame. After welding, the screeds are removed;

4. The edges of the corners, perpendicular to the plane of the wall, are cut off $50 \mathrm{~cm}$ from the edge of the frame;

5. Holes with a diameter of $30 \mathrm{~mm}$ for the passage of the tie bolts are drilled through the strips. Tieboltswith a diameter of $20 \mathrm{~cm}$ made of steel of class A-I are installed. After hanging the nuts, the latter are welded;

6. The separation wall is wrapped in a Rabitz mesh, which is attached with annealed wire to the frame elements;

7. After moistening the masonry, the separation wall is plastered with M-50 mortar. 


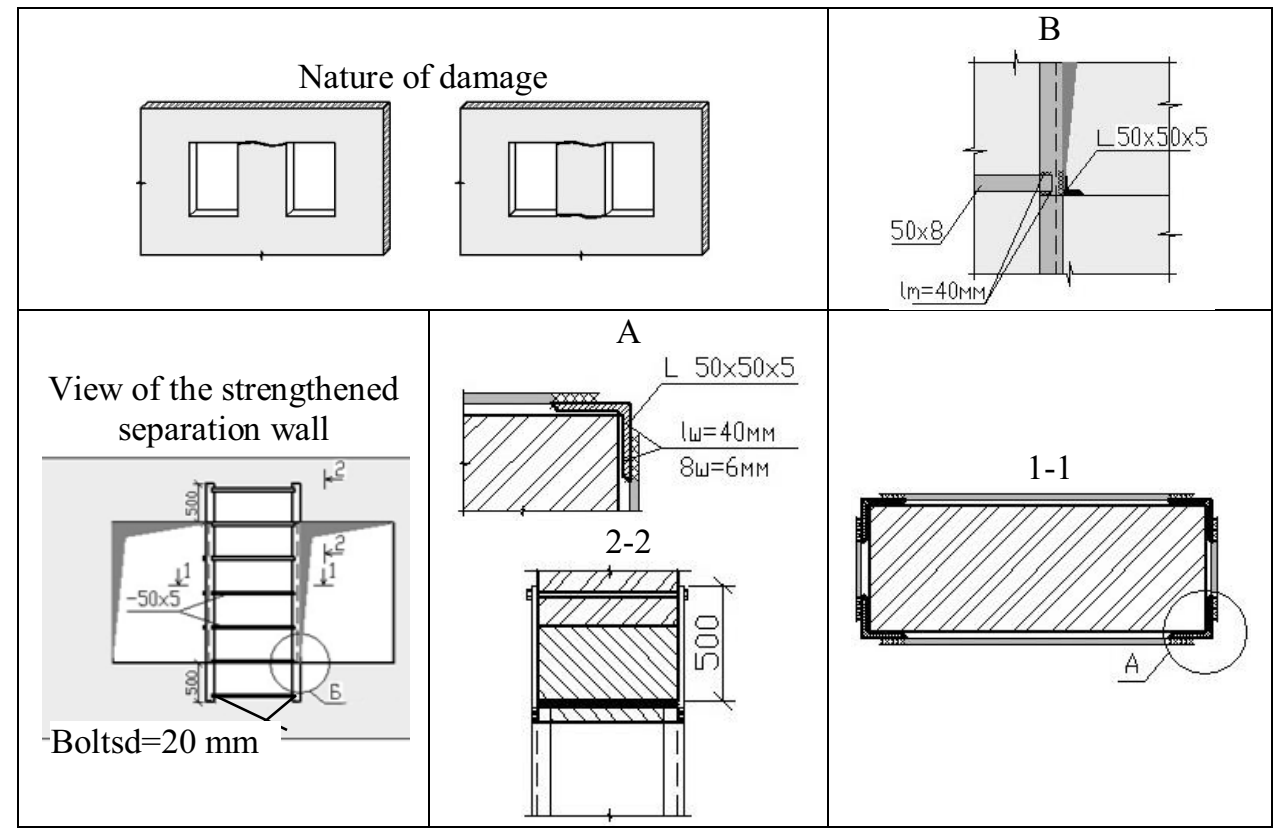

Fig. 3. Strengthening of separation walls with horizontal cracks.

\section{Separation walls with the loss of individual sections.}

Area of application.

1. In separation walls with local insignificant indents (Fig. 4).

2. If the masonry is chipped (no more than half).

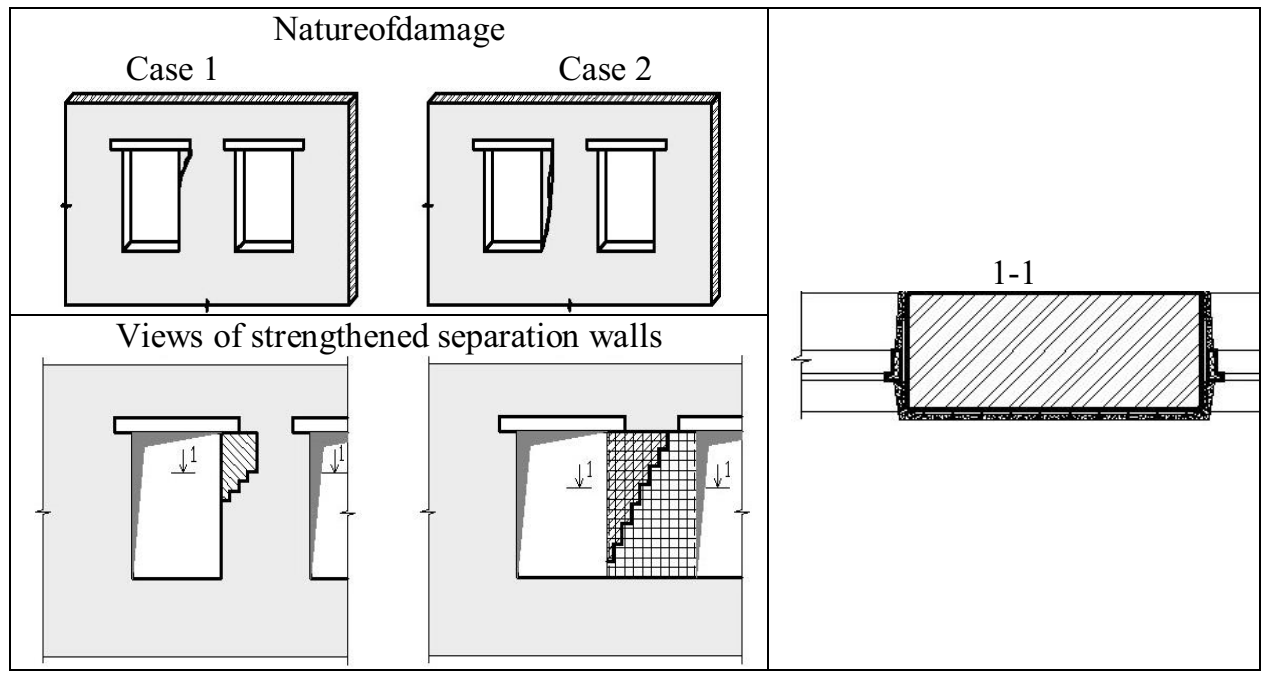

Fig. 4. Strengthening of separation walls with local minor indents and when the masonry is chipped (no more than half).

List of technological operations:

\section{Case I}

1. Support the lintel with a temporary post; 
2. Dismantle the destroyed section of the masonry and re-lay it using cement mortar; Case II

1. Remove the plaster and rinse the wall with water under pressure;

2. Support the lintel with a temporary post;

3. Dismantle the destroyed section of the masonry and re-lay it using cement mortar;

4. A grid with a mesh size of $150 \times 150 \mathrm{~mm}$ from a wire with a diameter of $5 \mathrm{~mm}$ is installed and fixed with the help of nails $\mathrm{L}=100$, driven into the seams of the masonry;

5. Plastering above the grid with M-50 mortar.

The specified strengthening of the separation wall can be replaced by a metal frame device (see Fig. 1 and 2) or by a complete re-laying of the wall.

\section{Strengthening of walls}

\section{Walls with diagonal cracks}

Area of application.A diagonal crack inclined at the junction of the walls or anywhere else (Fig. 5).

List of technological operations:

1. Plaster is removed from both sides of the wall;

2. The crack is cleared;

3. The seams are cleaned in both directions to a depth of $1.0-1.5 \mathrm{~cm}$;

4. The surface of the cleaned wall is washed with water under pressure;

5. A grid with a mesh size of $150 \times 150 \mathrm{~mm}$ from a wire with a diameter of $5 \mathrm{~mm}$ is installed. In other cases, the grids should be installed by analogy with the image in this drawing, observing the specified dimensions from the edge of the grid to the crack equal to $50 \mathrm{~cm}$;

6. The grids are connected using Z-shaped anchors $d=6 \mathrm{~mm}$ made of steel of class $\mathrm{A}-1$, installed in pre-drilled holes $\mathrm{d}=20 \mathrm{~mm}$, in a checkerboard pattern every $60-80 \mathrm{~cm}$;

7. The grids are fixed at a distance of $1.0 \mathrm{~cm}$ from the wall;

8. Plastering above the grid with M-50 mortar;

9. The drilled holes are carefully filled with mortar.

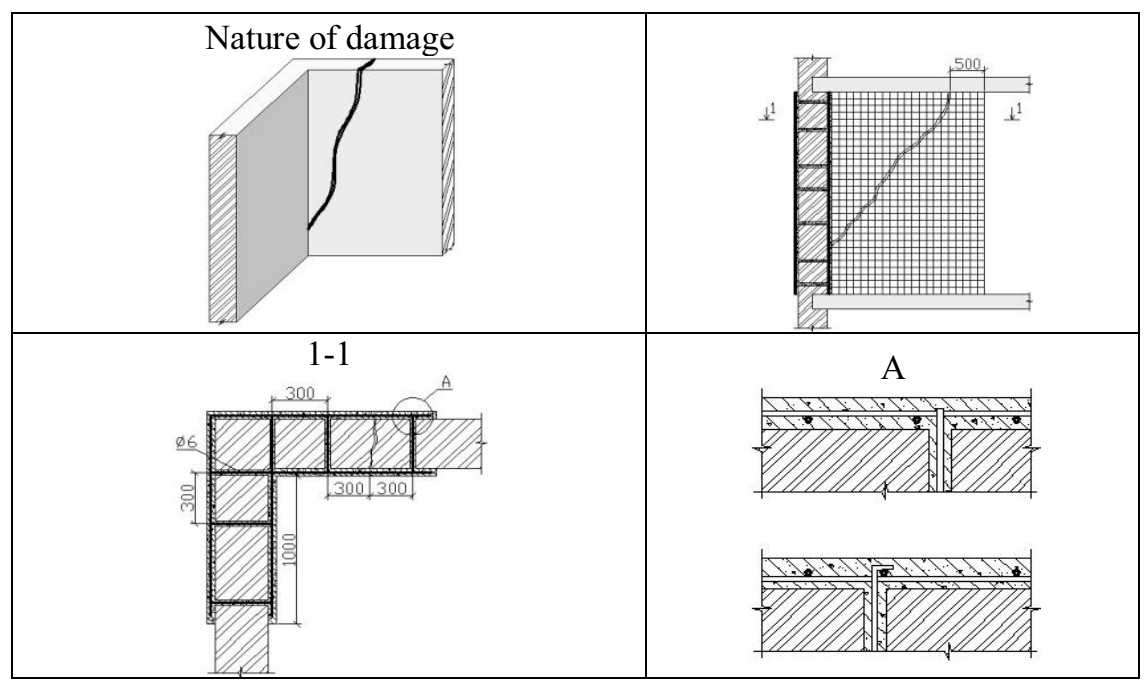

Fig. 5. Strengthening of walls with diagonal cracks inclined at the junction of the walls or anywhere else. 
Walls with diagonal, $x$-shaped, horizontal and chaotic cracks

Area of application. Diagonal, cruciform, horizontal and chaotic cracks (Fig. 6).

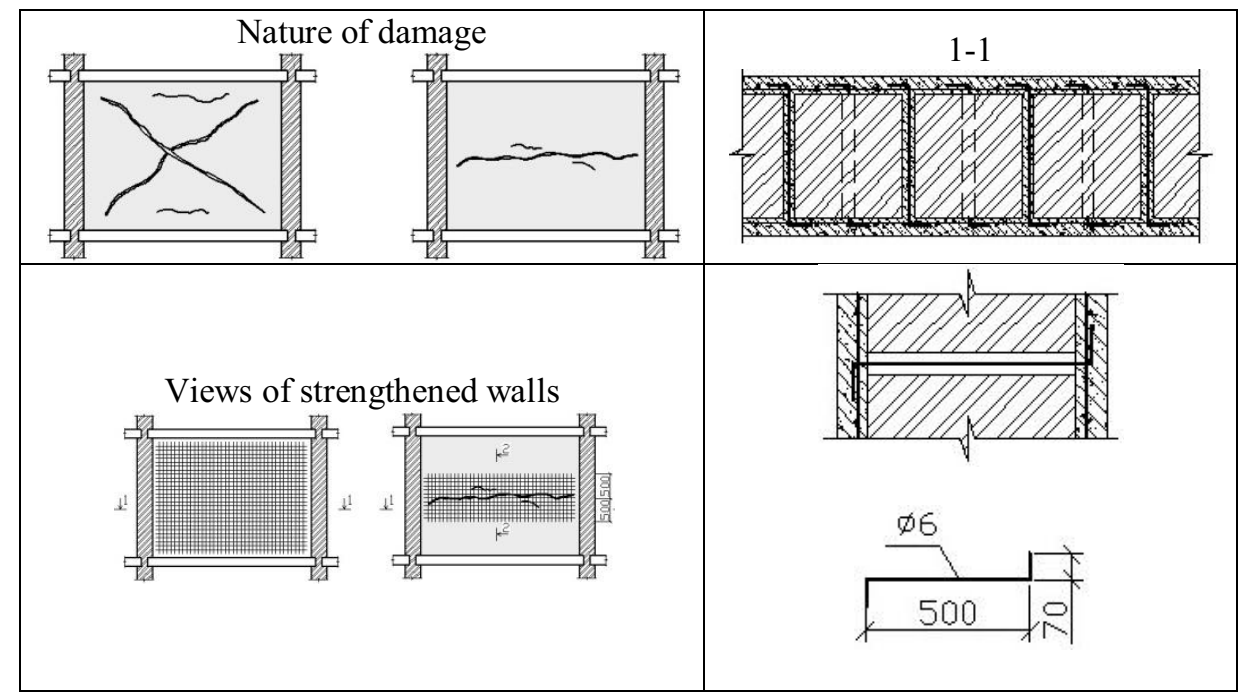

Fig. 6. Strengthening of walls with diagonal, cruciform, horizontal and chaotic cracks

List of technological operations:

1. Plaster is removed from both sides of the wall;

2. The crack is cleared;

3. The seams are cleaned in both directions to a depth of $1.0-1.5 \mathrm{~cm}$;

4. The surface of the cleaned wall is washed with water under pressure;

5. A grid with a mesh size of $150 \times 150 \mathrm{~mm}$ from a wire with a diameter of $5 \mathrm{~mm}$ is installed;

6. The grids are tied by means of Z-shaped anchors with a diameter of $6 \mathrm{~mm}$ made of steel of class A-I in previously drilled holes $\varnothing 20 \mathrm{~mm}$ in a staggered manner every $60-80$ $\mathrm{cm}$;

7. Before shotcreting, the walls are heavily moistened;

8. The grids are fixed at a distance of $1 \mathrm{~cm}$ from the wall;

9. The drilled holes are carefully filled with mortar;

10. The walls are shotcreted. The thickness of the plaster should be $30-40 \mathrm{~mm}$.

\section{Walls with diagonal cracks at lintel supports and doorways}

Area of application.

In case of damage to individual transverse walls with openings (Fig. 7). 


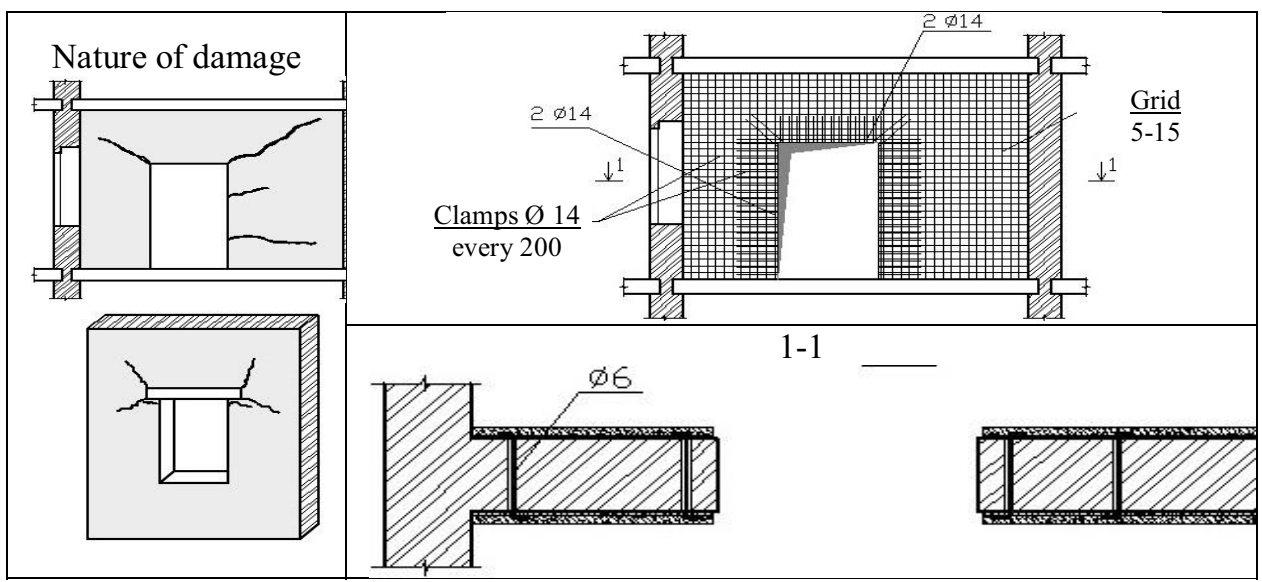

Fig. 7. Strengthening in case of damage to individual transverse walls with openings.

List of technological operations:

1. All plaster on both sides of the wall is cleaned, and horizontal and vertical joints are cleaned to a depth of $1.0-1.5 \mathrm{~cm}$;

2. To install the anchors, holes with a diameter of $15-20 \mathrm{~mm}$ are drilled in the wall in a checkerboard pattern with dimensions of $60-80 \mathrm{~cm}$;

3. The surfaces of the cleaned walls are washed with water under pressure;

4. A grid with a mesh size of $150 \times 150 \mathrm{~mm}$ from a wire with a diameter of $5 \mathrm{~mm}$ is installed on both sides of the wall. Z-shaped anchors are installed in the holes and connected to the grid. The distance of the grid from the wall is $1.0 \mathrm{~cm}$. The opening is framed with reinforcement with a diameter of 14-16 mm, which is connected by open clamps with a diameter of 5-6 mm between themselves and the reinforcing grid;

5. The walls are shotcreted. The thickness of the plaster must be at least $30-40 \mathrm{~mm}$. Before shotcreting, the walls are heavily moistened. Mortar grade for shotcreting is not lower than M-100;

6. The overlap of the wall at the welded joint must be at least $200 \mathrm{~mm}$.

\section{Strengthening of wall abutments}

\section{Strengthening with partial separation of walls}

Area of application. Partial separation of the wall vertically (Fig.8).

List of technological operations:

1. Holes with a diameter of $30 \mathrm{~mm}$ for straps and for tie bolts are drilled in height after 70 $\mathrm{cm}$;

2. External channel bars No. 10 are installed with pre-drilled holes for bolts and ties;

3. The channel bars are fastened to the outer wall with tie bolts, with the installation of metal gaskets with dimensions of 100x100x10 mm from the inner surface of the wall;

4. Overlays with a section of 50x5 $\mathrm{mm}$ are welded to the channel bars;

5. Ties made of class A-I reinforcement with a diameter of $20 \mathrm{~mm}$ are passed through the drilled holes and fastened along the detail of unit "B" with the installation of locknuts.

The holes in which the bolts are inserted are jetted;

6. Fastenings on the outer surface of the wall should be plastered over the grid;

7. Seal the crack with cement mortar;

8. All welded seams $\mathrm{L}_{\mathrm{s}}=50 \mathrm{~mm}, \delta_{\mathrm{s}}=6 \mathrm{~mm}$. 


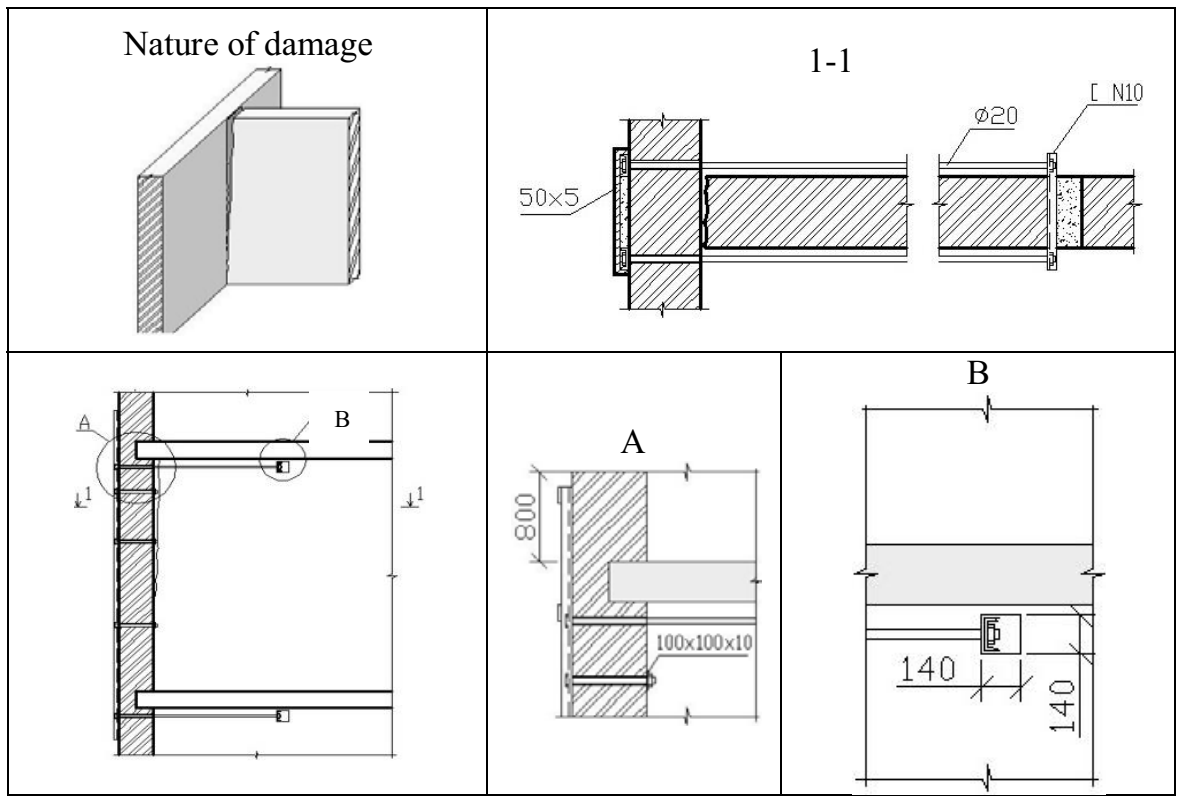

Fig. 8. Strengthening with partial separation of walls vertically.

\section{Strengthening with full separation of the wall vertically}

Area of application. Full separation of the wall vertically (Fig. 9).

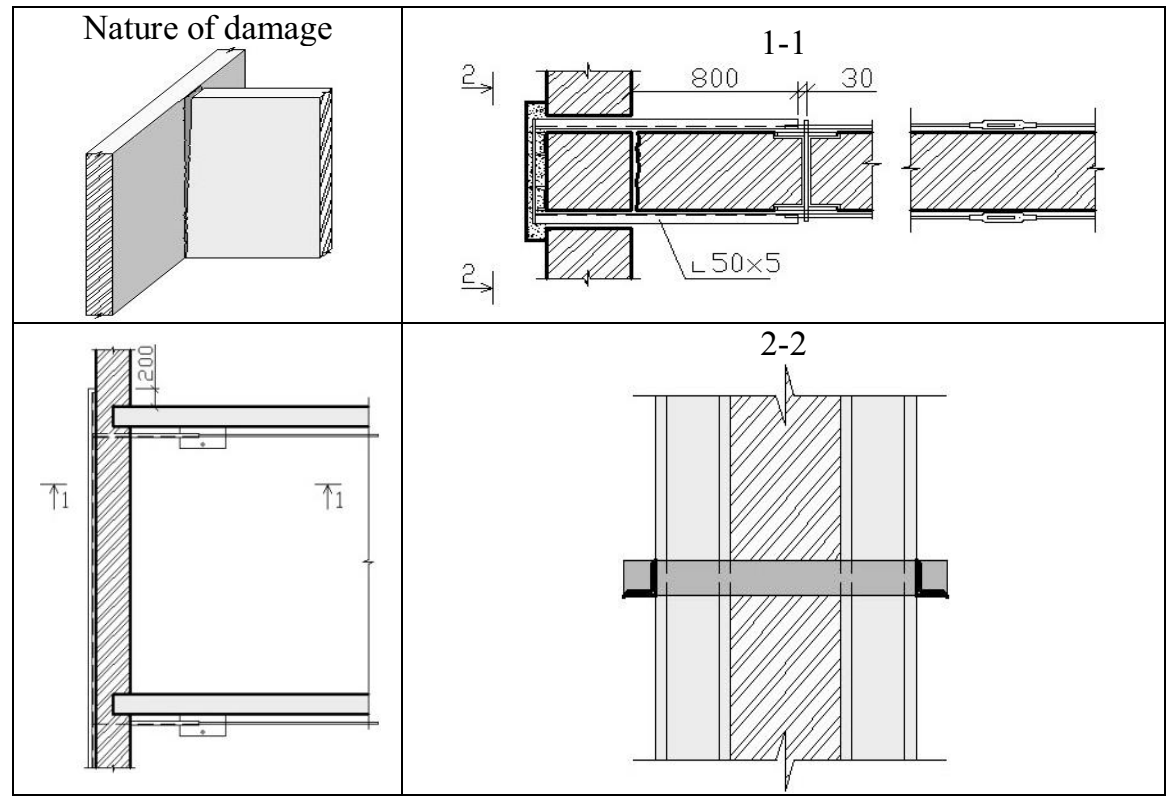

Fig. 9. Strengthening with full separation of walls vertically.

List of technological operations:

1. Holes of $65 \times 65 \mathrm{~mm}$ are punched, holes with $\mathrm{d}=30 \mathrm{~mm}$ are drilled, and places for the plate (pos. 1) with dimensions of $200 \times 150 \times 10$ are prepared. Top of holes $65 \times 65 \mathrm{~mm}$ is at a distance of $10 \mathrm{~cm}$ from the bottom of the floor; 
2. Corners $50 \times 50 \times 5$ are installed;

3. Channel bars No. 10 are installed and welded to the corners;

4. Channel bars $50 \mathrm{~cm}$ in height are welded with strips of 50x5 made of strip steel;

5. Plates (pos. 1) are installed and tightened with bolts;

6. Ties from reinforcement $\varnothing 30 \mathrm{~mm}$ are welded to the upper flanges of the corners with two welded seams $\mathrm{L}_{\mathrm{s}}=70 \mathrm{~mm}, \delta_{\mathrm{s}}=6 \mathrm{~mm}$.

7. The tensioning is carried out using tensioning devices (jacks, couplings, etc.) or by heating round strands. The degree of tension is determined by ensuring a tight fit of the channel bars to the wall, but with heating of not more than $300^{\circ} \mathrm{C}$. When the specified degree of tensioning is reached, the corners are welded to plate 1 with a double-sided seam $\mathrm{L}_{\mathrm{s}}=70 \mathrm{~mm}, \delta_{\mathrm{s}}=6 \mathrm{~mm}$;

8. In case of creation of tension force by heating method, welding of strands is performed only on one side. The second side is welded after reaching the required elongation;

9. Crack and holes for passing corners are cleaned and jetted;

10. From the outside, the protruding elements are plastered along the grid.

\section{Strengthening of lintels}

\section{Reinforced concrete lintels with cracks}

Area of application. When strengthening lintels with cracks with opening in the tensioned zone up to $4 \mathrm{~mm}$ (Fig.10).

List of technological operations:

1. Plaster is cleaned along the entire length of the lintel on both sides

2. The lower horizontal seams within the lintel supports are cleaned from both sides to a depth of $6 \mathrm{~cm}$. Holes are punched in the seam between the lintel and the overlying masonry

3. In the cleared seams, 50x5 corners are placed using the mortar and temporary screeds

4. In the holes punched above the lintel, 50x5 strip iron is laid

5. Corners are welded with horizontal overlayingstrips 50x5

6. Strengthened lintel is plastered with M-50 mortar

7. When the lintel is shifted, the support areas and the plane of abutment to the masonry are jetted

8. In case of significant destruction of the lintel and the masonry above it, it is advisable to disassemble the masonry and replace the lintels

9. Welded seams $\mathrm{L}_{\mathrm{s}}=50 \mathrm{~mm}, \delta_{\mathrm{s}}=6 \mathrm{~mm}$

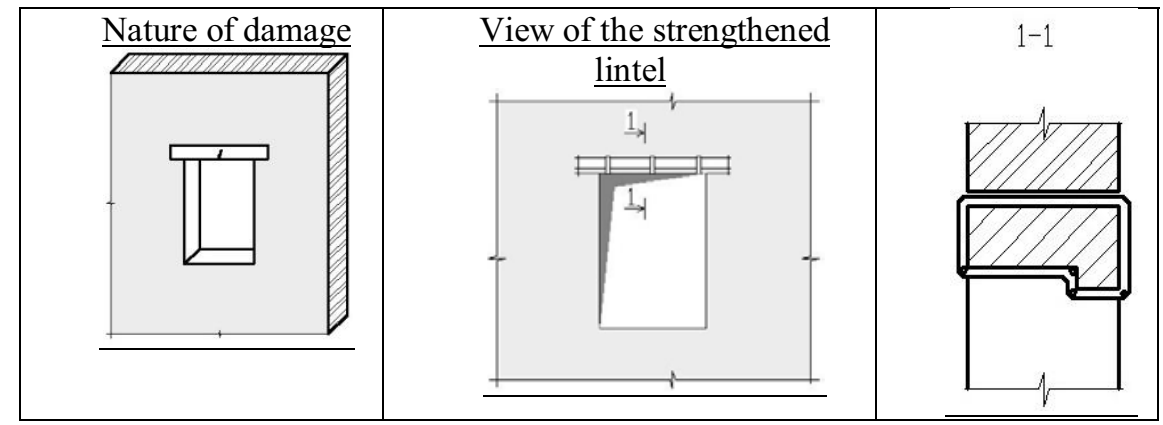

Fig. 10. Strengthening of cracked reinforced concrete lintels

When strengthening lintels with cracks with opening in the stretched zone up to $4 \mathrm{~mm}$ and a distance from the opening to the overlap of at least $100 \mathrm{~cm}$, it is recommended to strengthen according to Fig. 11. 
List of technological operations:

1. The plaster is removed in the area above the level of the top of the deformed lintel by 50 $\mathrm{cm}$;

2. A $21 \mathrm{~cm}$ high indent is punched at a distance of $35 \mathrm{~cm}$ from the top of the existing lintel. The depth of the indent is $13 \mathrm{~cm}$, the length is $50 \mathrm{~cm}$ longer than the existing opening;

3. In the brickwork, 3 holes $\mathrm{d}=30 \mathrm{~mm}$ are drilled through the indents;

4. The masonry and indent cleaned of plaster are thoroughly rinsed with water under pressure;

5. Channel bars with drilled holes are installed and fastened with bolts $\mathrm{d}=12 \mathrm{~mm}$ made of steel of class A-1;

6. Indents are plastered with M-50 mortar;

7. In case of significant destruction of the lintel and masonry above it, it is advisable to disassemble the masonry and replace the lintels;

8. Before the production of works, the ceiling is supported by temporary poles.

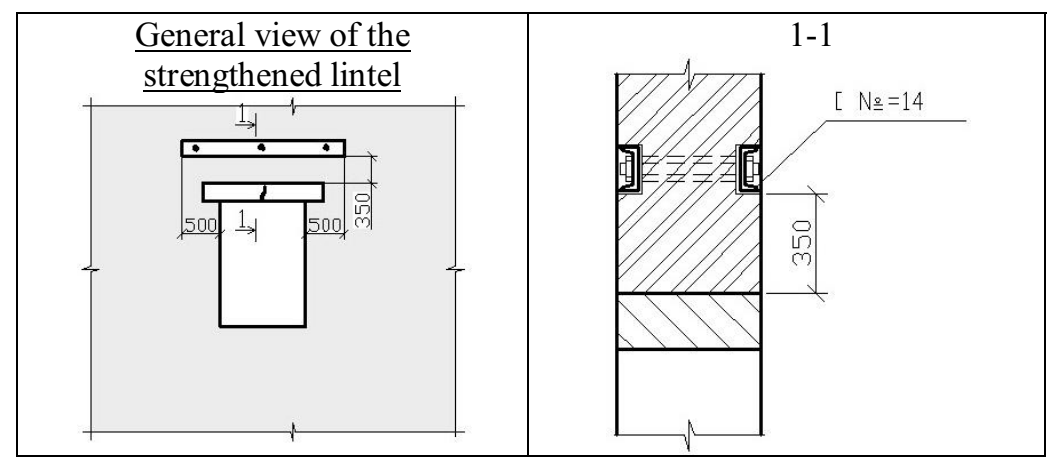

Fig. 11. Strengthening of reinforced concrete lintels with faults

\section{Brick wedge-shaped lintels}

Area of application. Strengthening of brick wedge-shaped lintels is performed in the presence of cracks and in the absence of damage to the overlying masonry (Fig. 12).

If the masonry above the lintel does not exceed $1.0-1.5 \mathrm{~m}$, it is advisable to disassemble the masonry and replace the lintel with a bar-shaped one.

List of technological operations:

1. Clear the horizontal seam of the lintel on one side to a depth of $7 \mathrm{~cm}$;

2. In the cleared seam, lay a corner $75 \times 75 \times 8$ on cement mortar so that there is no gap between the corner and the lintel, as well as between the corner and the wall;

3. At a distance of $35 \mathrm{~cm}$, holes $\mathrm{d}=15 \mathrm{~cm}$ are punched on both sides of the opening. The bottom of the holes is at the level of the top of the opening;

4. The horizontal seam at the bottom of the lintel on the other side is cleared to a depth of $7 \mathrm{~cm}$, and the corner $75 \times 75 \times 8$ is laid;

5. Cut corners $75 \times 75 \times 8$ are placed in the holes punched along the edges of the opening and welded to the main corners. Height of welded seams $\delta=6 \mathrm{~mm}$;

6. The area of the hole is hammered with hard concrete on non-shrinking cement;

7. At the edges of the opening and in the middle, strips of $50 \times 6 \mathrm{~mm}$ are weldedto the underside of the corners;

8. When the walls are not plastered, the metal parts are painted 2 times;

9. With plastered walls, a Rabitz mesh is welded to the corners and plastered;

10. Welded seams $L_{s}=50 \mathrm{~mm}, \delta_{\mathrm{s}}=6 \mathrm{~mm}$. 


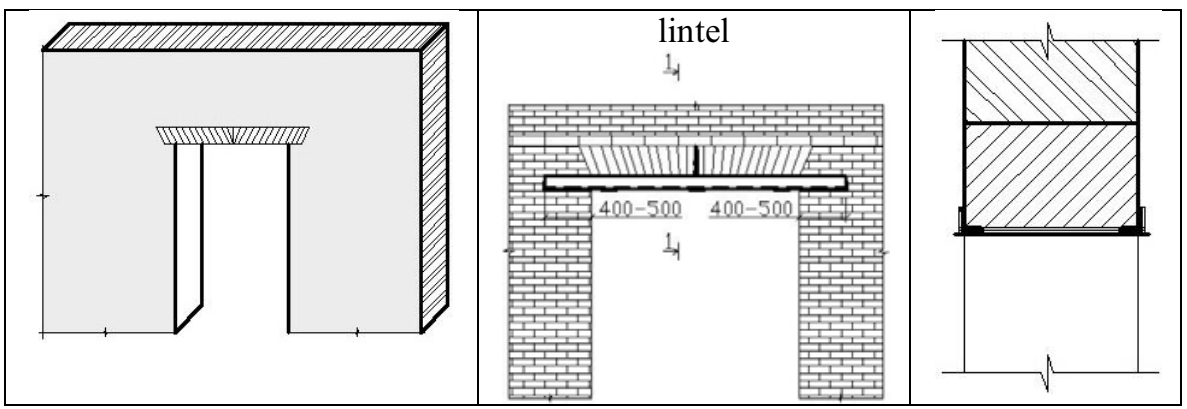

Fig. 12. Strengthening of brick wedge-shaped lintels.

\section{Destroyed arched and lancet lintels (with an opening width of up to $1.0 \mathrm{~m}$ ).}

Area of application. When strengthening the arched and lancet lintels with openings up to $100 \mathrm{~cm}$ wide (Fig. 13).

List of technological operations:

1. The plaster is cleaned in the area of deformed masonry on both sides of the lancet lintel, and the horizontal and vertical joints are cleared to a depth of $1.0-1.5 \mathrm{~cm}$;

2. The cleaned masonry surface is washed with water under pressure;

3. A welded grid with a mesh size of $150 \times 150 \mathrm{~mm}$ from a wire with a diameter of $5 \mathrm{~mm}$ is applied on both sides of the heavily moistened and washed masonry. The grid is tied to nails $\mathrm{L}=100 \mathrm{~mm}$ hammered into the seams of the masonry. The grid is installed at a distance of $1 \mathrm{~cm}$ from the wall with an overlap of the skewback of at least $25 \mathrm{~cm}$;

4. The grid is cut along the circular contour of the opening;

5. Shotcreting should be done. The thickness of the shotcreted plaster must be at least 3-4 $\mathrm{cm}$. Before shotcreting, the masonry must be heavily moistened. The grade of mortar must be at least M-100.

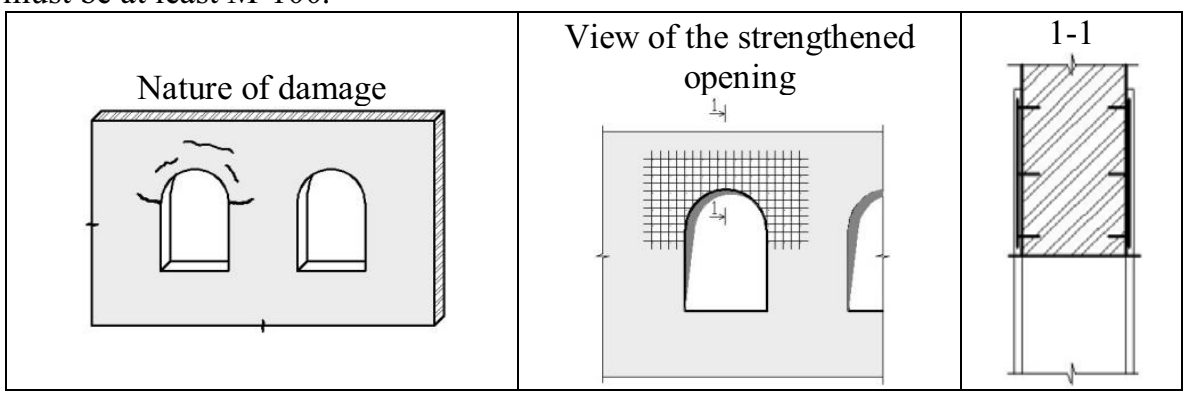

Fig. 13. Strengthening of arched and lancet lintels with openings up to $1 \mathrm{~m}$ wide.

\section{Construction and strengthening of anti-seismic belts}

\section{Strengthening of the anti-seismic reinforced concrete belt in the presence of cracks}

Area of application.

Strengthening of reinforced concrete belts in the presence of cracks more than $3 \mathrm{~mm}$ wide at the intersection of the longitudinal and transverse belts (Fig. 14).

\begin{tabular}{|l|l|l|l|}
\hline View of & $\underline{1-1}$ & $\underline{2-2}$ & $\underline{3-3}$ \\
\hline
\end{tabular}




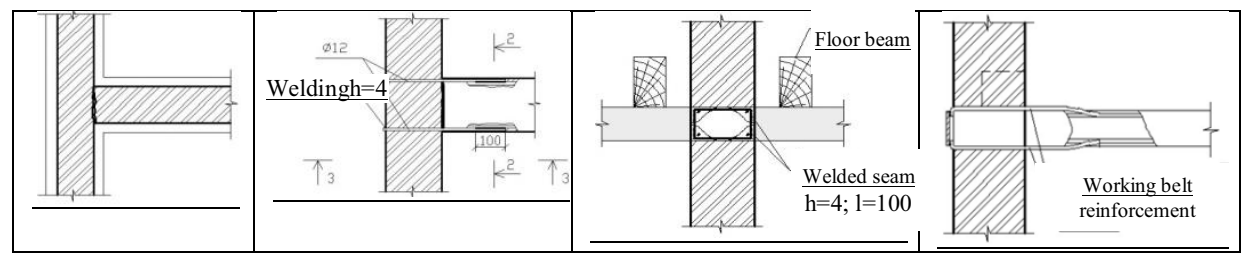

Fig. 14. Strengthening of reinforced concrete belts in the presence of cracks more than $3 \mathrm{~mm}$ wide at the intersection of the longitudinal and transverse belts.

List of technological operations:

1. 4 holes $\mathrm{d}=20 \mathrm{~mm}$ are punched in the places where the masonry adjoins the belt;

2. At a distance of $20-30 \mathrm{~cm}$ from the junction plane, the belt reinforcement is exposed;

3. Punched holes and exposed concrete are washed with water;

4. Rods $d=12 \mathrm{~mm}$ made of steel of class A-1 are installed, bent along the welding points and welded;

5. From the outer side of the wall, the rods are welded with steel plates $\mathrm{d}=12 \mathrm{~mm}$;

6. Punched holes are sealed with mortar; the exposed area is concreted;

7. Parts protruding outside are plastered with M-50 mortar.

\section{Arrangement of a metal anti-seismic belt}

Area of application.

Multi-storey buildings with wooden floors in the absence of seismic belts (Fig. 15).

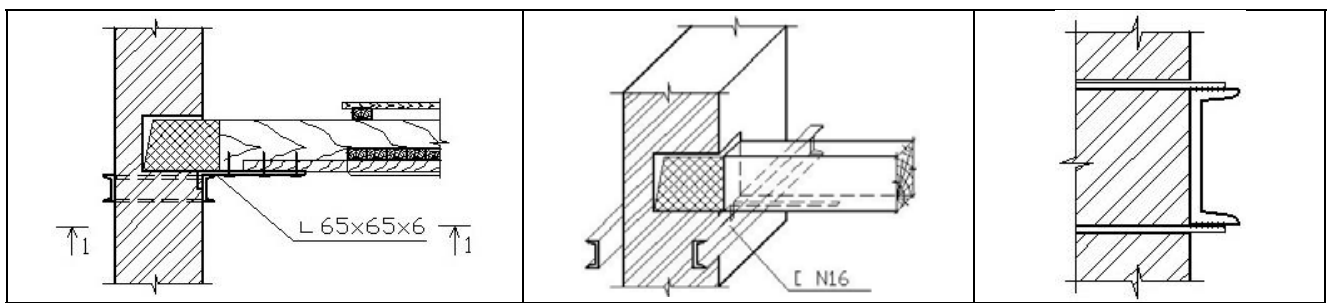

Fig. 15. Arrangement of a metal seismic belt in buildings with wooden floors in the absence of belts.

List of technological operations:

1. The ceiling is disassembled to a width of $1 \mathrm{~m}$ along the perimeter of the walls;

2. Plaster is removed from both sides of the walls in the places where the channel bars are installed;

3. Holes are punched at the level of the bottom of the beam for the $50 \times 5$ strip; strip length is measured locally;

4. Strips are inserted into the punched holes, and channel barNo. 10 is inserted between them;

5. Strips are welded to the outer channel bar;

6. Fasteners from a 50x5 strip are installed along the axis of the floor beams;

7. The wall is compressed with channel bars;

8. Strips are welded on the second side;

9. Fasteners are sewn to the floor beams with the help of brushes;

10. Punched holes are jetted;

11. At the intersection of the walls, the channel bars from below and from above are welded with gussets with a leg size of $25 \mathrm{~cm}$. The dimensions of the outer gusset are to be determined locally. Welding is performed with an intermittent seam. The total length of the seam is not less than $150 \mathrm{~mm}$ on one side; the thickness of the gusset is $10 \mathrm{~mm}$; 
12. All welds are $6 \mathrm{~mm}$ thick.

Arrangement of reinforced concrete anti-seismic belt.

Area of application. Strengthening of multi-storey buildings with wooden floors in the absence of anti-seismic belts (Fig. 16).

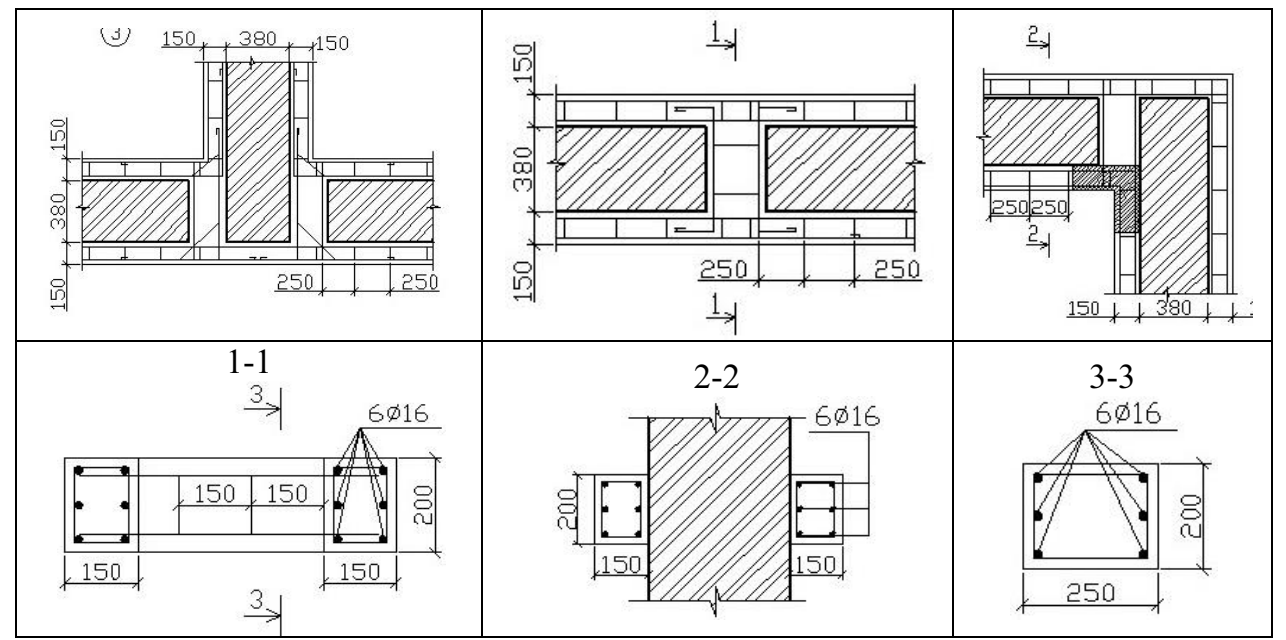

Fig. 16. Arrangement of reinforced concrete anti-seismic belt (buildings with wooden floors in the absence of this belts).

List of technological operations:

1. The ceiling and floor are disassembled to a width of $1 \mathrm{~m}$ along the perimeter of the walls;

2. Plaster is removed from both sides of the walls along the overlap $50 \mathrm{~cm}$ wide;

3. The vertical and horizontal seams of the masonry are cleared to a depth of $1.0-1.5 \mathrm{~cm}$;

4. Holes are punched in the masonry with dimensions of $20-25 \mathrm{~cm}$ for connecting dowels;

5. Cleaned surfaces and punched holes are rinsed with water under pressure;

6. Reinforcement cages are installed, and concreting of dowels and belts is carried out on heavily moistened masonry;

7. In the absence of embedding of wooden beams in the wall, it is necessary to ensure their anchoring with a belt;

8. Concrete of class not lower than B7.5

\section{Strengthening of gables, parapets, cornices and canopies}

\section{Restoration of destroyed gables, parapets and cornices}

Area of application. Diagonal horizontal cracks in gables, parapets, eaves, gable walls or partial collapse.

List of technological operations:

1. All gables, parapets, cornices and gable walls made of brickwork, regardless of the degree of their damage, except for those made with a reinforced concrete frame (with vertical and horizontal reinforcement elements), are recommended to be disassembled;

Before dismantling the brickwork of the gable walls, the brick support posts for the girders are replaced with wooden ones, with the installation of braces;

2. Replacement of destroyed gables should be carried out using wooden or light metal 
frame structures;

3. The cladding of the frame of the gables should be made of modern lightweight durable materials, depending on the architectural significance.

The canopy frame is made in a wooden version or using light metal frame structures. The lower rail is attached to the wall with metal anchors or wire grids placed every $1.5-2.0$ $\mathrm{m}$. The frame cladding is made of modern lightweight durable materials, depending on the architectural significance.

Note. During the reconstruction of buildings of historical, cultural and architectural significance, the reinforcement of gables, parapets, cornices and canopies is carried out without disassembling them according to individual projects.

\section{Strengthening of partitions}

\section{Strengthening of partitions}

Area of application. Disruption of the connection between the partition and the walls, without loss of stability (Fig.17).

List of technological operations:

1. The partition is temporarily strengthened. Skirting boards and anchor ties are disassembled;

2. On both sides of the partition up to 3 meters long, horizontal bars of $60 \times 60 \mathrm{~mm}$ are installed on the top and at the bottom, fastened with nails $\mathrm{L}=100 \mathrm{~mm}$ between themselves and to the wooden floor;

It is possible to install metal corners $50 \times 50 \mathrm{~mm}$, which are fastened with overlapping with dowels V-7 or nails $\mathrm{L}=100 \mathrm{~mm}$ (Fig. 18);

3. When the partition is more than $3 \mathrm{~m}$ long, the bars are additionally installed in the vertical direction. The bars are painted with oil paint;

It is possible to fasten the partition to the walls in a vertical plane with fasteners;

Partitions up to 3 meters long are attached only with anchors to the walls;

4. After fastening the partitions, the temporary fasteners are removed;

5. In the case of the option of fastening the partition with corners, the latter are painted with oil paint 2 times;

6. In case of loss of stability, the partition is disassembled and laid again.

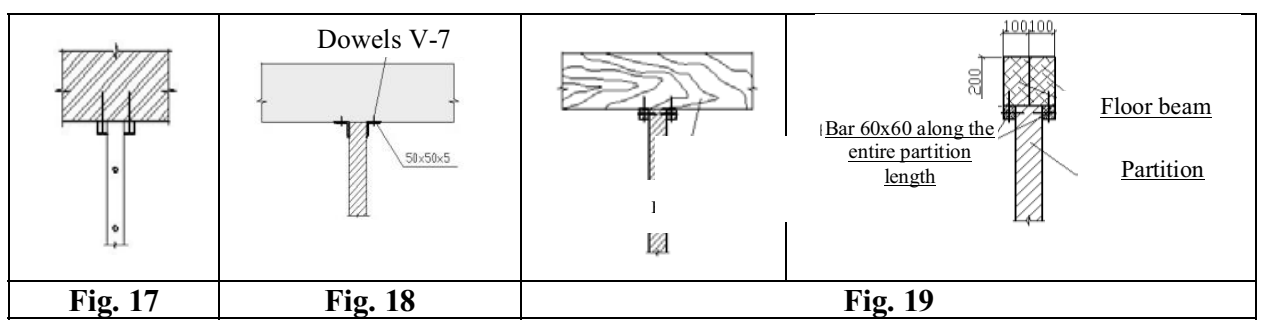

\section{Results}

Thus, methods and guidelines for the technology of work (including their composition, features and sequence of implementation) have been developed for the restoration and strengthening of residential and civil buildings with walls made of fired bricks, which received various damages as a result of earthquakes.

The above methods can also be used when strengthening the structures and elements of 
existing buildings of the noted design solution, certain parameters of which do not meet the requirements of the currently valid republican design standards (KMK) [1].

In addition, for each of the considered cases and strengthening options, appropriate standards have been developed in relation to the labor intensity and cost of performing work, the recommended composition of links and the corresponding standard set (due to existing limits on the volume of the paper, they are not describedhere).

\section{Discussion}

The results obtained, in the form of developed methods and technologies for the production of works, formed the basis of a special "Manual", the preparation of which is being completed by the authors by now. In the "Manual" presented in this paper, the materials are reflected in more detail, cover other possible cases and situations encountered in practice, illustrated in detail and supplied with sets of working drawings made in the AutoCAD environment. In particular, it contains methods, technologies and technical regulation of the issues of strengthening of other elements and structures (reinforced concrete, wooden and metal), which also take place in buildings of the considered design solution.

It should be noted that in Uzbekistan, there are still no specialized regulatory documents (ShHK) regulating the technology of work to strengthen structures. In this regard, these standards have been drawn up on the basis of an appropriate selection of types of processes and works, which are suitable and similar in nature and meaning, regulated by the current domestic ShHKfor repair and other types of work [2-4, etc.], as well as the corresponding selection from foreign regulatory documents [5-6, etc.]. In this regard, the compiled standards require an appropriate (more detailed) substantiation and adjustment, which is the subject of further research and development in this area. The task of developing and approving a set of relevant standard method statements is also set.

It should also be noted that work on strengthening structures is technologically peculiar, delicate, and unique and requires the manufacturer to have appropriate training and professionalism, the availability of appropriate tools, devices and equipment, which is included in the concept of a standard set.

\section{Conclusions}

The methods, technologies and technical regulation of the issues of restoration and strengthening of elements and structures of brick buildings presented in the paper and in the noted "Manual" are recommended for use by design, construction and operating organizations, private developers, etc.

\section{References}

1. KMK2.01.03-19 Building in seismic areas (The ministry of building of RUz, Tashkent, 2019).

2. ShHK 4.02.61-07 Collections of resource budget norms on it is repair-civil work. Plaster works (The State committee on architecture and building RUz, Tashkent, 2007).

3. ShHK4.02.69-07 Collections of resource budget norms on it is repair-civil work. Other it is repair-civil work (The State committee on architecture and building RUz, Tashkent, 2007).

4. ShHK4.02.53-07 Collections of resource budget norms on it is repair-civil work. The collection of 53 "Walls" (The State committee on architecture and building RUz, 
Tashkent, 2007).

5. Khan Shahzada, Muhammad Javed, Bashir Alam, MansoorKhan, ZaighamAli, Hassan Khan and Syed Shahan Ali Shah, Strengthening of Brick Masonry Walls against Earthquake Loading International Journal of Advanced Structures and Geotechnical Engineering 01 (01) (2012). ISSN 2319-5347.

6. Lei Zhu and Congfeng Sun, New Low-interference Seismic Strengthening Method for Masonry Structures Journal of Engineering Science and Technology Review 11 (2) $143-152(2018)$. 